\title{
A Concept Analysis of Clinical Reasoning in Occupational Therapy
}

\author{
Sho Maruyama ${ }^{1,2}$, Satoshi Sasada ${ }^{3}$, Yohei Jinbo ${ }^{4}$, Peter Bontje ${ }^{5}$ \\ ${ }^{1}$ Department of Occupational Therapy, Graduate School of Human Health Sciences, Tokyo Metropolitan University \\ ${ }^{2}$ Department of Rehabilitation, Shonan-Keiiku Hospital \\ ${ }^{3}$ Department of Occupational Therapy, School of Rehabilitation, Kanagawa University of Human Services \\ ${ }^{4}$ Department of Occupational Therapy, Chigasaki-Rehabilitation College \\ ${ }^{5}$ Department of Occupational Therapy, School of Health Science, Tokyo Metropolitan University
}

\begin{abstract}
Introduction: Acquiring competence in clinical reasoning is regarded as key to meet the challenges of integrating knowledge into one's practice. Learners and educators need a shared understanding of what is clinical reasoning because they need appropriate means for reflection and feedback. However, the clinical reasoning concept needs revisiting in the current context, which is becoming increasingly diverse and complex. The purpose of this study was to analyse the clinical reasoning concept and develop a common framework.

Methods: Rodgers' concept analysis was selected for this theoretical study. Accordingly, a systematically search of multiple databases yielded 903 articles and 135 articles that met the inclusion criteria were extracted, and of these 30 articles randomly sampled for analysis. Data analysis was done following Rodgers' method of thematic analysis.

Results: The attributes were four 'therapeutic thinking processes' and two 'professional thinking skills.' The application contexts were 'clinical and non-clinical practice settings,' and 'professional education.' Moreover, the antecedents were 'professional factors' and 'practice factors,' and the consequences were 'professional developments' and 'professional attitudes and behaviours.'

Conclusion: The resulting framework of clinical reasoning can be the basis for developing pedagogies and assessment of clinical reasoning competence acquisition.
\end{abstract}

Keywords: allied health occupations, clinical competence, clinical reasoning, concept analysis, occupational therapy

(Asian J Occup Ther 17: 17-25, 2021)

\section{Introduction}

Clinical reasoning $(\mathrm{CR})$ in occupational therapy is a core-ability [1] that occupational therapists and students develop with clinical experience [2]. Acquiring competence in CR is regarded as key to meet challenges of integrating knowledge into one's practice in the changing practice contexts [3]. CR has become key now students and practitioners need to learn to deal with challenges such as meeting expectations of clients who are better informed than in the past; adapting to increasing diversity in the delivery of services and practical contexts outside the traditional institutional settings; and how to

Received: 6 May 2020, Accepted: 29 September 2020

Corresponding to: Sho Maruyama, Rehabilitation dept., ShonanKeiiku Hospital, 4360 Endo, Fujisawa-city, Kanagawa, Japan e-mail: ot1985sho@gmail.com

(C2021 Japanese Association of Occupational Therapists master an increasing body of knowledge [4].

However, CR includes tacit knowledge embedded into the experience of a practice, which is difficult to verbalise and to teach [5]. More recently, educators and learners alike started considering $\mathrm{CR}$ as an intersubjective process in which educators and learners co-construct CR [6]. For these reasons, they need a shared understanding of what $\mathrm{CR}$ is. Previous studies attempted to clarify what CR might be and its types through mainly qualitative researches [5, 7]. However, while scholars have re-conceptualized these understanding, the CR concept has not been sufficiently confirmed, expanded, or refuted through empirical studies [8].

In addition, Unsworth and Baker [9] argued that developing a standardized assessment scale is urgently needed. Developed in the past, the Self-Assessment of Clinical Reasoning and Reflection (SACRR) and the California Critical Thinking Skills Test (CCTST) were designed for the assessment of students' reasoning and 
reflection skills $[10,11]$. However, these two scales were not developed based on the CR concept in occupational therapy. Also, the items of SACRR and CCTST are limited to scientific reasoning aspects of CR. Since a review of research literature did not reveal any other assessment tools, it appears that no valid, reliable assessments of CR exist [12]. Therefore, in addition to educational reasons, it is necessary to fully establish the recent evolving understandings in the profession regarding $\mathrm{CR}$ the future development of valid assessment methods [13].

Adding further complexity to this lack of clarity on how to operationalise and assess CR, some have started to reframe the concept in terms of professional reasoning and therapeutic reasoning $[14,15]$. Professional reasoning broadens the $\mathrm{CR}$ concept to include the reasoning that occurs in non-medical environments, such as schools and community settings, as well as including reasoning done by occupational therapy teachers, clinical educators, and occupational therapy managers as they conceptualise their practice [15]. However, despite this proposed new terminology, research has not progressed on clarifying different features of CR concept. These evolving insights about the CR concept lend further support to the need to revisit the concept in the context of current occupational therapy practices that are becoming more diverse and complex [12]. In this paper, we will use the term $\mathrm{CR}$, albeit including the professional and therapeutic reasoning that occurs in the non-traditional diversifying practice contexts, being mindful of the intersubjective process in which educators and learners co-construct CR [6].

In conclusion, based on the uncertainties in how $\mathrm{CR}$ is understood, we argue there is a need for analysing the concept of CR. While CR in occupational therapy is typified as scientific, narrative, pragmatic, ethical, interactive, and conditional reasoning [15], there is a need for developing a common framework that not only integrates the different types of CR, but one that also explains how CR occurs. Analysing the CR concept is essential, not only for supporting educators and learners in the development of learners' CR skills but clarifying the concept is also fundamental to the future development of assessment tools of CR. It is well known that concept analysis is well suited to such a double challenge [16]. Accordingly, the purposes of this study were to analyse the concept of CR and to develop a common framework that integrates the different types of CR in occupational therapy.

\section{Methods}

\section{Study design}

Rodgers' concept analysis [17] was selected for the following reasons [18]. First, the assumption of Rogers' methodology that concepts are dynamic lends itself to understanding the concept of $\mathrm{CR}$, which is sensitive to temporal changes [8]. Secondly, Rodgers' methodology is an interpretive method and, particularly suited to study the CR concept, which explains processes taking place in persons' minds [5]. Finally, Rodgers' concept analysis provides a strategy suited for more fully explaining how concepts occur by identifying and defining their essential components, antecedents and consequences, and application contexts $[16,17]$.

\section{Data gathering}

Data gathering consisted of systematically searching and screening for relevant literature [17]. Search terms were 'occupational therapy,' and 'clinical reasoning,' or surrogate terms of 'professional reasoning,' 'therapeutic reasoning,' and 'narrative reasoning.' The searched databases were PubMed, Cumulative Index to Nursing and Allied Health Literature (CINAHL), Health Source, SocINDEX, Education Resources Information Center (ERIC), OTseeker, Ichushi (Japan Medical Abstracts Society), J-STAGE (Japan Science and Technology Agency) and Medical online, using advanced search processes. The searches spanned the years 1983-2017, because CR as a concept was introduced into occupational therapy in 1983 [19]. The first author (SM) accessed the databases on October 23, 2017.

Figure 1 shows the flow for identifying and screening extracted papers in line with the PRISMA guidelines [20]. From the 903 studies identified, 504 were omitted because of double selection. A further 264 papers were excluded after titles and abstracts were screened for the following criteria: (a) the subject of articles is not CR; (b) not reported by occupational therapists; (c) not related to occupational therapy practice and education. Rodgers [17] suggests that $20 \%$ or about 30 articles of the retrieved literature be included in the sample. Accordingly, with 135 papers remaining, we randomly sampled 30 by Excel's random number table (see Table 1).

\section{Data analysis}

Thematic analysis was conducted in the following interactive back-and-force process: generating initial codes, searching for themes, reviewing themes, generating a thematic map of the analysis, and generating names for each theme [17,21]. First coding the data was guided by using the following questions: What are the features of the concept? What factors are preceding the concept? What are the significant consequences of the concept? Moreover, what kind of situation and scope does the concept apply?

Subsequently, we identified (a) attributes and appli- 


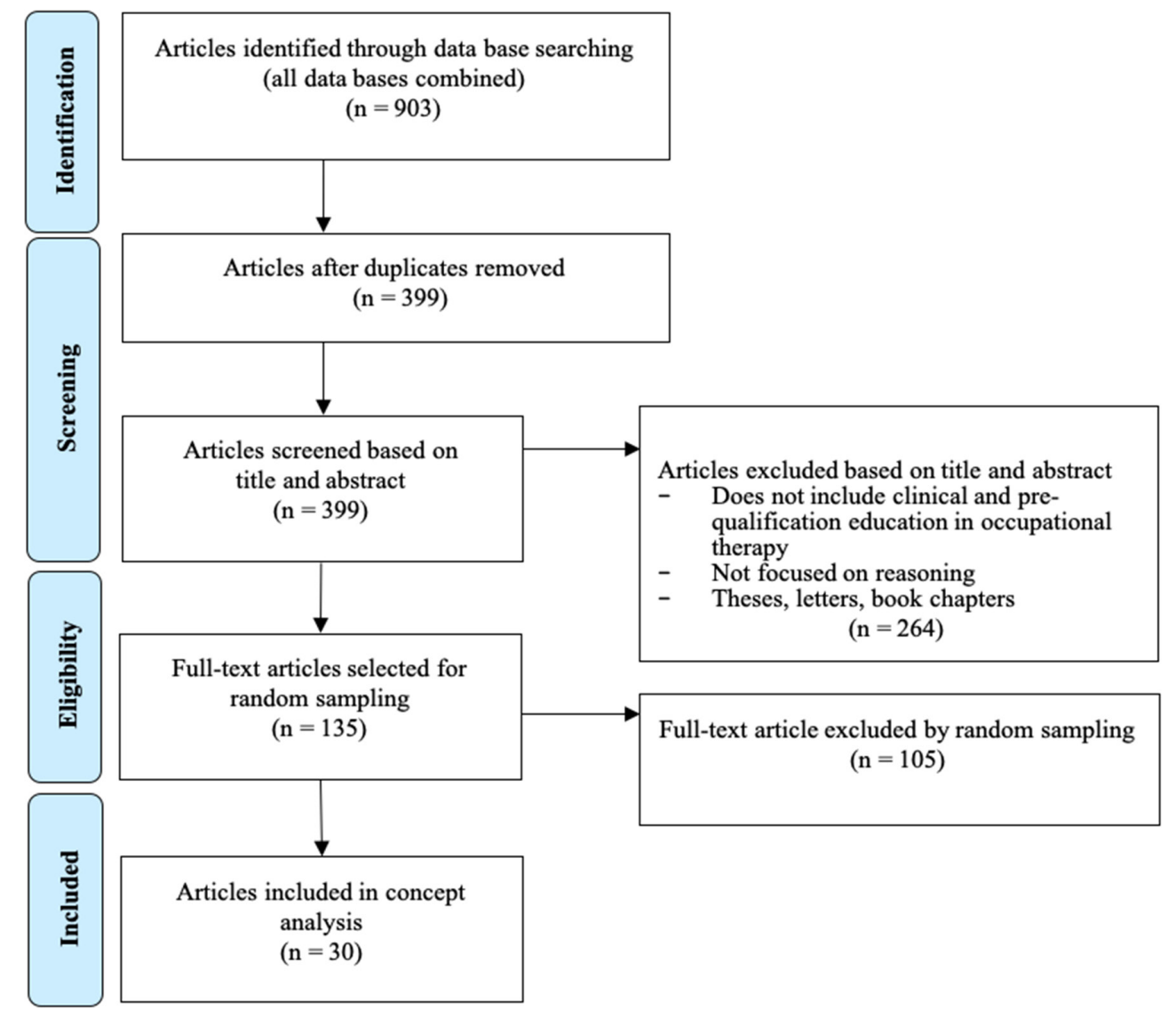

Fig. 1. The number of studies identified and screened for concept analysis. Diagram flow refers to Preferred Reporting Items for Systematic Reviews and Meta-Analyses (PRISMA) and data gathering methods of Rodgers' concept analysis.

cation contexts, (b) antecedents, and (c) consequences of the concept. (a) Attributes and application contexts comprised of the definition of the concept. (b) Antecedents were multiple conditions, behaviours, or attitudes that occurred before the concept. (c) Consequences were events or phenomena that occurred afterwards to the concept. This phase of analysis was an inductive coding process of continually organizing and reorganizing data until a cohesive, comprehensive, and relevant system of descriptors was generated [17]. MAXQDA 2018 (VERBI) software was used to support the qualitative data analysis. Besides, to reduce the bias of the authors (SM, YJ, PB) doing the analysis, records of the data analysis were audited by an author (SS) of this study team who was not involved in the actual data analysis.

\section{Results}

We clarified the attributes, preconditions, results, and application context of the concept through Rodgers' concept analysis of the CR literature. The attributes were four 'therapeutic thinking processes' and two 'professional thinking skills,' the application contexts were 'clinical and non-clinical practice settings' and 'professional education of occupational therapists (pre-qualification and continuing education).' Moreover, the antecedents were 'professional factors' and 'practice factors,' and the consequents were 'professional developments' and 'professional attitudes and behaviours' (see Figure 2). The numbers inserted in brackets in the below text correspond to numbers of the analysed articles listed in Table 1.

\section{Attributes}

\section{Therapeutic thinking processes}

'Therapeutic thinking processes' were an essence of CR in occupational therapy, in which occupational therapy practitioners and students used to organise and support clinical thinking [42]. It included the following four therapeutic thinking processes: (a) scientific evidence-driven thinking process, (b) professional ethicsdriven thinking process, (c) practical contexts-driven thinking process, and (d) client's narrative-driven thinking process. Practitioners and students used these four types of therapeutic thinking processes together in their 
Table 1 List of articles included for the analysis

[26] Gee BM, Strickland J, Thompson K, Miller LJ. Exploring the influence of an e-learning sensory processing-based module for graduate level occupational therapy students on clinical reasoning: A pilot study. Occupational Therapy International. 2017; 24: 1-8.

[27] Unsworth C, Baker A. Systematic review of professional reasoning literature in occupational therapy. British Journal of Occupational Therapy. 2016; 79(1): 5-16.

[28] Robertson D, Warrender F, Barnard S. The critical occupational therapy practitioner: How to define expertise? Australian Occupational Therapy Journal. 2015; 62(1): 68-71.

[29] Shafaroodi N, Kamali M, Parvizy S, Mahraban AH, O'toole G. Factors affecting clinical reasoning of occupational therapists: a qualitative study. Medical Journal of the Islamic Republic of Iran. 2014; 28(19): 50-9.

[30] Kristensen HK, Borg T, Hounsgaard L. Aspects affecting occupational therapists' reasoning when implementing research-based evidence in stroke rehabilitation. Scandinavian Journal of Occupational Therapy. 2012; 19(2): 118-31.

[31] Parkinson S, Shenfield M, Reece K, Fisher J. Enhancing professional reasoning through the use of evidence-based assessments, robust case formulations and measurable goals. British Journal of Occupational Therapy. 2011; 74(3): 148-52.

[32] Coker P. Effects of an experiential learning program on the clinical reasoning and critical thinking skills of occupational therapy students. Journal of Allied Health. 2010; 39(4): 280-6.

[33] Mu K, Coppard BM, Bracciano A, Doll J, Matthews A. Fostering cultural competency, clinical reasoning, and leadership through international outreach. Occupational Therapy in Health Care. 2010; 24(1): 74-85.

[34] Carrier A, Levasseur M, Bedard D, Desrosiers J. Community occupational therapists' clinical reasoning: identifying tacit knowledge. Australian Occupational Therapy Journal. 2010; 57(6): 356-65.

[35] Taylor B, Robertson D, Wiratunga N, Craw S, Mitchell D, Stewart E. Using computer aided case-based reasoning to support clinical reasoning in community occupational therapy. Computer Methods and Programs in Biomedicine. 2007; 87(2): 170-9.

[36] Falk-Kessler J, Ciaravino EA. Student reflections as evidence of interactive clinical reasoning skills. Occupational Therapy in Health Care. 2006; 20(2): 75-88.

[37] Scaffa ME, Wooster DM. Effects of problem-based learning on clinical reasoning in occupational therapy. The American Journal of Occupational Therapy. 2004; 58(3): 333-6.

[38] Mitchell R, Unsworth CA. Role perceptions and clinical reasoning of community health occupational therapists undertaking home visits. Australian Occupational Therapy Journal. 2004; 51(1): 13-24.

[39] Leicht SB, Dickerson A. Clinical reasoning, looking back. Occupational Therapy in Health Care. 2002; 14(3-4): 105-30.

[40] Lyons KD, Crepeau EB. Clinical Reasoning of an Occupational Therapy Assistant. The American Journal of Occupational Therapy. 2001; 55(5): 577-81

[41] Gibson D, Velde B, Hoff T, Kvashay D, Manross PL, Moreau V. Clinical reasoning of a novice versus an experienced occupational therapist: a qualitative study. Occupational Therapy in Health Care. 2000; 12(4): 15-31.

[42] Neistadt ME. Teaching clinical reasoning as a thinking frame. The American Journal of Occupational Therapy. 1998; 52(3): 221-9.

[43] Fisher GS. Health care reform, manage care, and clinical reasoning: implications for ethical occupational therapy practice. Occupational Therapy in Health Care. 1997; 11(1): 27-37.

[44] Neistadt ME. Teaching strategies for the development of clinical reasoning. The American Journal of Occupational Therapy. 1996; 50(8): 676-84.

[45] Munroe H. Clinical reasoning in community occupational therapy. British Journal of Occupational Therapy. 1996; 59(5): $196-202$.

[46] Alnervik A, Sviden G. On clinical reasoning: patterns of reflection on practice. The Occupational Therapy Journal of Research. 1996; 16(2): 98-110.

[47] Roberts AE. Clinical reasoning in occupational therapy: Idiosyncrasies in content and process. British Journal of Occupational Therapy. 1996; 59(8): 372-6.

[48] Creighton C, Dijkers M, Bennett N, Brown K. Reasoning and the art of therapy for spinal cord injury. The American Journal of Occupational Therapy. 1995; 49(4): 311-17.

[49] Schell BA, Cervero RM. Clinical reasoning in occupational therapy: An integrative review. The American Journal of Occupational Therapy. 1993; 47(7): 605-10.

[50] Burke JP, DePoy E. An emerging view of mastery, excellence, and leadership in occupational therapy practice. The American Journal of Occupational Therapy. 1991; 45(11): 1027-32.

[51] Mattingly C. The narrative nature of clinical reasoning. The American Journal of Occupational Therapy. 1991; 45(11): 998-1005.

[52] Mattingly C. What is clinical reasoning? The American Journal of Occupational Therapy. 1991; 45(11): 979-86.

[53] Fleming MH. Clinical reasoning in medicine compared with clinical reasoning in occupational therapy. The American Journal of Occupational Therapy. 1991; 45(11): 988-96.

[54] Cohn ES. Fieldwork education: Shaping a foundation for clinical reasoning. The American Journal of Occupational Therapy. 1989; 43(4): $240-4$.

[55] Rogers JC. Eleanor Clarke Slagle Lectureship-1983; clinical reasoning: the ethics, science, and art. The American Journal of Occupational Therapy. 1983; 37(9): 601-16. 


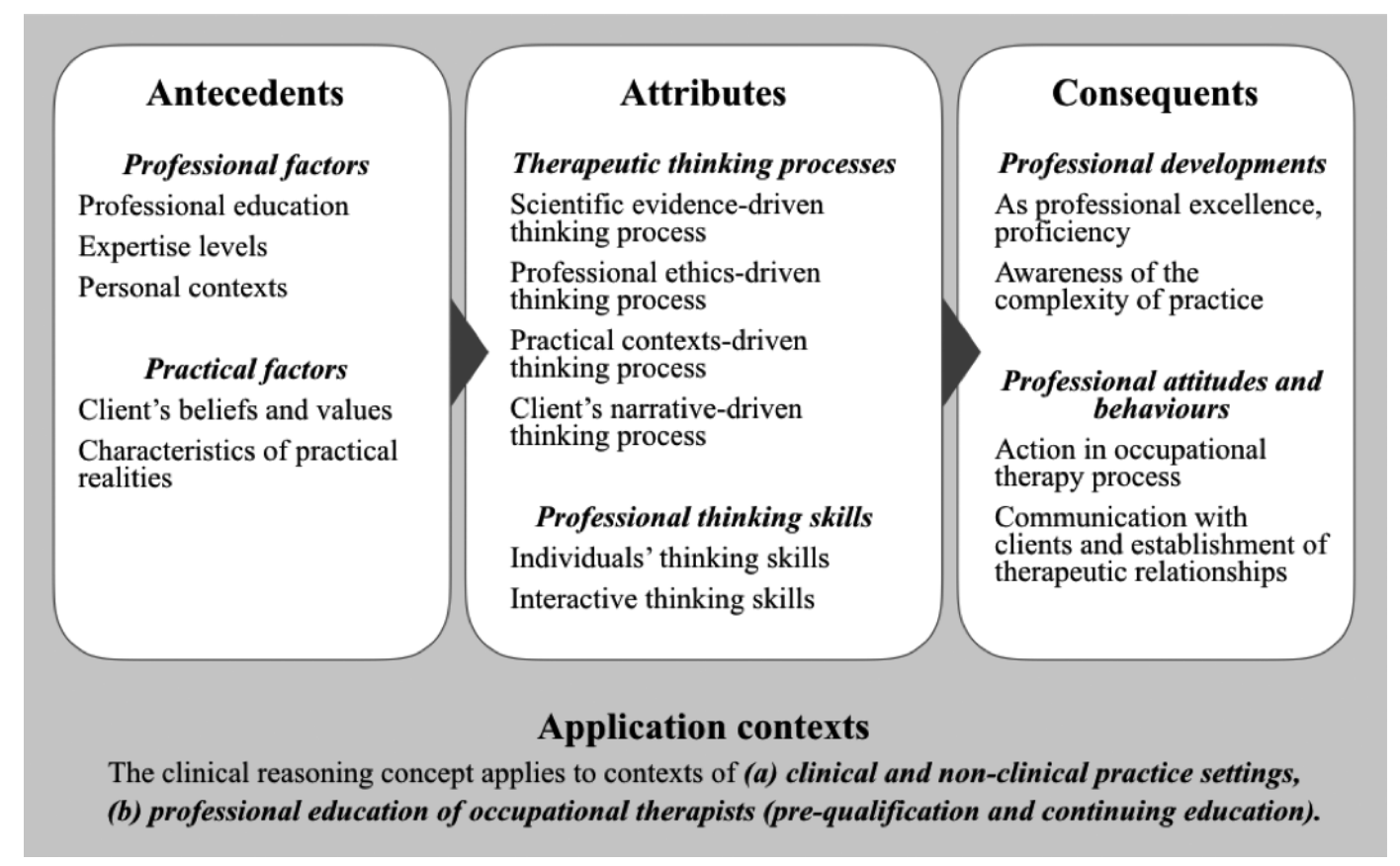

Fig. 2. Result of concept analysis of clinical reasoning in occupational therapy

daily practice, amounting to complex, multifaceted, and dynamic thinking processes $[34,38]$.

The scientific evidence-driven thinking process. Practitioners and students used the scientific evidencedriven thinking process to inform problem-definition, problem-analysis, and problem-solving in practice $[28$, $49,53,55]$. This therapeutic thinking process was considered to professionalise occupational therapy practice [49], because it included a logical process based on hypothesis formulation and testing [55], facilitated systematic information gathering and interpretation [53], and selecting the best intervention to address clients' performance problems [42]. The scientific evidencedriven thinking process, conventionally called scientific reasoning, included diagnostic and procedural reasoning as used in medicine $[49,53]$. Also, it was often mainly relied on by novice practitioners. The scientific evidence-driven thinking process reflected the scientific paradigm of the profession. In the modern occupational therapy paradigm, practitioners and students focused on the health condition, activity level/balance, and participation in daily occupations and/or occupational performance of clients $[39,44,53]$.

The professional ethics-driven thinking process. Practitioners and students used the professional ethicsdriven thinking process for focusing not on 'what could be done' in a therapy session, but on 'what should be done' [55] to determine priorities for execution in prac- tice where a myriad of potential ethical dilemmas exists [43]. They used this therapeutic thinking process to find solutions when they were challenged with multiple ongoing and complex issues [43], or when clinical data contradicted their expectations and experience $[43,54]$. A more recent phenomenon, the professional ethicsdriven thinking process was used for decisions of professional ethics, because of changes within the structure of health care service delivery (e.g., the need to a focus on reducing costs), with competing interests of the health care system (e.g., prioritise the care given to clients), right of clients, and complicated choice of treatment (e.g., balance of benefit and risk) [39, 43]. Thus, the professional ethics-driven thinking process included dilemma recognition, dilemma analysis, self-monitoring to given standards and norms, and self-reflection [28]. Finally, it was used by practitioners and students to manage the influences of their personal contexts (e.g., values, knowledge, experiences, skills, and routines) [34, $39,42,49]$.

The practical contexts-driven thinking process. Practitioners and students used the practical contextsdriven thinking process to manage the influences of practical contexts when considering the therapeutic possibilities within a given occupational therapy setting $[42,48,49]$. Practical contexts included such aspects as time resources (e.g., time available for therapy sessions, an overall length of therapy programs, a therapist's schedule), space resources (e.g., physical environment, 
availability of equipment and tools, client familiarity with space), and social resources (e.g., social support network, health care insurance, rules within the facility) $[34,39,42,49]$. These factors were the starting point of this thinking process. The practitioners and students used this therapeutic thinking process to integrate considerations of practical contexts into their decisions about intervention decisions or recommendations to clients $[48,49]$.

The client's narrative-driven thinking process. Practitioners and students used the client's narrative-driven thinking process when understanding clients as occupational beings and interpreting stories told by clients. They used this therapeutic thinking process to focus on the clients' life story and the meanings of occupations to clients. The client's narrative-driven thinking process facilitated creating prospects for and with clients and often together with clients' families [49, 51-53], to promote participation of clients and their families in occupational therapy $[49,53]$, and to revise interventions to meet the clients' needs $[42,44,46]$. In other words, occupational therapy students and practitioners used this therapeutic thinking process when they were focused on understanding clients' temporality (their past, present, and future), client's subjectivity and inter-subjectivity between clients and their families [42, 46, 49, 52, 53].

\section{Professional thinking skills}

'Professional thinking skills' of practitioners and students were core skills of occupational therapy practice $[29,30]$, and these skills were composed of (e) individual's thinking skills and, (f) interactive thinking skills.

Individuals' thinking skills. Practitioners and students used one's individual thinking skills for synthesizing or combining types of therapeutic thinking processes $[45,46,49]$, which in the analysed articles were also referred to as mental manipulation or information processing skills $[42,45]$. Differences in individuals' thinking skills were related to occupational therapists' accumulation of clinical experience [27, 41, 44], enhancing their various thinking directions (inductive and deductive approaches) and range, depth, precision, and speed of reasoning [39, 41, 55]. For example, while novice therapists were able to describe their CR, it appeared to be confined to the scientific thinking process [41]. Also, students could note clients' concerns but lacked an in-depth understanding of how disability had an impact on the life of individuals [47]. On the other hand, experienced therapists frequently weaved subjective and intersubjective perspectives into their understanding of clients [47], and they used intuitive thinking and complex reasoning types [39, 41, 45, 53]. Also, experienced therapists shifted rapidly and effortlessly from one form of CR to another (e.g., scientific to narrative reasoning) $[44,53]$.

Interactive thinking skills. Practitioners and students used the interactive thinking skills to plan, conduct and reflect on their practice $[26,50,52,55]$. The interactive thinking skill was considered essential to interact with and better understand clients during face to face encounters [50]. It was 'know-how' that therapists used in the conduct of their work and how they derived a 'best' course of action with a client [38]. For example, experienced practitioners facilitated the delivery of meaningful occupational therapy experiences for clients through communication and collaboration with clients and other professionals in complex practice situations $[35,39,42$, 46]. Establishing these effective relationships were considered crucial to the success of the occupational therapy process $[39,50]$. Besides, this thinking skill was based on individual knowledge and tacit knowledge, because it was also based on tacit understanding and habitual knowledge gained through experience [26, 41, 52].

\section{Application contexts}

The CR concept applied to clinical and non-clinical occupational therapy practice settings that include schools, workplaces, and communities. Practitioners and students used the CR concept as the language for the professional education of occupational therapists (pre-qualification education, continuing education). For example, using a CR-frame to organise clinical observations was an effective way to help entry-level occupational therapy students learn and apply the CR concept $[42,54]$. Also, it was said that understanding and articulation of CR were desirable to facilitate the learner and educator communication and sharing of knowledge [28]. Thus, CR was recognised as having significant implications for occupational therapy practice and education $[27,41,49]$. However, the scope of the CR concept was limited to occupational therapy for clients as individuals and groups, but its application to practices for populations was not included in the analysed articles.

\section{Antecedents}

Antecedent included professional factors of occupational therapists and practical factors (see Figure 2). The professional factors included professional education, occupational therapists' expertise levels and personal contexts. Professional education included e-learning, 
experiential learning, and reflections in/on occupational therapy practice, which enhanced CR skills $[26,32,36$, 37]. Expertise levels included the knowledge and use of knowledge, perceived capability, and accumulated clinical experiences in specific areas [33, 34, 39, 41]. For example, CR was influenced by individuals' experiences, which different models of intervention with similar clients [55]. Expertise levels which related to the clinical experience in a specific area, influenced the types of CR preferred and the depth of understanding [38, 54]. Personal contexts included motivation, self-efficacy, beliefs, values routine, use of theory, and therapists' paradigms/world views [34, 39, 41, 49]. These personal contexts might affect the specific types of $\mathrm{CR}$ used individually, or of multiple CR types simultaneously [34]. The thinking processes used thus highly reflected personal contexts [27, 34].

The practical factors included clients' beliefs and values, relationships between clients and occupational therapy practitioners (or students), and characteristics of practical realities $[29,34]$. Thus, CR was neither context-free nor value-free because it was influenced by a wide range of environmental, interpersonal, and intrapersonal factors and resources [45]. For example, factors affecting CR included client perceptions of what constitutes 'good therapy' [29]. The characteristics of practical realities included organizational, cultural, economic, social resources within occupational therapy practice $[29,34,49]$, such as the power relationships of occupational therapy within the organization, reimbursement resources for treatment services, and the kinds of available space and equipment [49]. The client-therapist relationship and interaction were another important antecedent of CR because this affected the client's active participation in the therapeutic process and the use of narrative and interactive reasoning [34].

\section{Consequences}

Consequences included professional development and enhanced professional attitudes and behaviours as occupational therapists (see Figure 2). Professional developments consisted of professional excellence and proficiency, and awareness of the complexity of professional practice. This was mainly reported as the growth and development of students and novice therapists or as being different between novices versus experts [27, 44]. The use of CR language helped them develop more precise thought processes sooner and provided a vocabulary for self-assessment and improvement of their CR skills [44], and to communicate and share knowledge $[28,44]$. Finally, CR helped to improve job satisfaction by making students and practitioners more aware of the complexity and depth of their work [44]. Thus, the usage of CR language was essential to professional development [50].

Professional attitudes and behaviours were included in the occupational therapy processes (e.g., implementation, modification, and explanation of occupational therapy plan, and collaboration with other professions), communication with clients in occupational therapy situations, and establishment of therapeutic relationships $[28,35,42,54]$. CR helped practitioners and students understand the meaning of illnesses and disorders to clients [42], and enabled them to see possibilities for creating essential experiences for the client and to build on these experiences by showing clients a possible future [51]. So, professional attitudes and behaviours facilitated meaningful occupational therapy experiences with clients [39]. In other words, the use of CR enhanced the perception of the effectiveness of interventions [28]. Besides, the use of CR language helped to explain the rationales behind therapists' decisions to clients, family members, team members, and insurance carriers [44], and it helped to negotiate complex practice issues [35].

\section{Discussion}

\section{A framework of the clinical reasoning concept}

In light of the three historical phases of CR studies, as classified by Márquez et al., the data included in this study covered the exploratory phase (7 articles), transition phase (10 articles), and consolidation phase (13 articles) [8]. Therefore, we argue that the results of this concept analysis are valid from a historical perspective of CR research. The results of Rodgers concept analysis, which identifies attributes ('therapeutic thinking processes' and 'professional thinking skills'), antecedents ('professional factors' and 'practice factors'), and consequences ('professional developments' and 'professional attitudes and behaviours') were identified (see Figure 2), taken together provide a framework for the CR concept that not only explains types of CR but also explains how CR occurs.

As one dimension of the CR framework this study identified $\mathrm{CR}$ attributes as four 'therapeutic thinking processes' (Figure 2). These are comparable to previous studies that identify the types of thinking process as answers to the question 'what is CR' [9]. Schell theorizes eight types (scientific, diagnostic, procedural, narrative, pragmatic, ethical, interactive, and conditional reasoning) as forms of CR [15]. Based on our analysis of literature we propose that our four types of CR (four types of thinking processes) as an authoritative classification of the various CR types.

Also, we clarified a new dimension of CR, namely 
the attributes 'professional thinking skills' (Figure 2). To the best of our knowledge this is at best under-reported in CR literature. That we could produce this result of thinking skills may be reflective of recent professional issues in occupational therapy such as evaluation of internationally developed competencies, and promoting awareness among members of the public and other professional groups of what the profession does [22]. In relation to this, previous studies describe the difference between novice and expert as a difference in the types of reasoning used by therapists [15], but not as a difference in skill. However, it is important to distinguish expert and novice in terms of skills in addition to 'what' experts and novices perform [23]. The identified CR individuals' and interactive thinking skills affords one an additional dimension to clarify the development of CR skills from novice to expert.

In addition, the professional and practical factors, the CR antecedents, not only affect individuals, but also these affect the quality of CR [24]. Also, we clarified the professional developments and professional attitudes/behaviours that are CR consequences. On the other hand, conventional CR research topics focused on 'what CR is' and 'what promotes CR' $[9,25]$. Thus, the antecedents and consequences identified in this study can be basic knowledge for informing educational approaches promoting the acquisition of CR.

Finally, the framework resulting from this study may be used to inform future research producing evidence of the efficacy of educational approaches and the authors are planning the development of a sufficiently reliable and valid assessment scale.

\section{Limitations}

The systematic search included English and Japanese articles, but because of random sampling by Rodgers' methodology [17], the analysed sample did not include Japanese articles (Table 2). However, as the number and scope of CR studies in Japan are still limited [12], we believe this did not affect the results of this study. Another methodological limitation was that we could not identify from the data, as per Rodgers' method for concept analysis [17], the exemplar as a typical example of CR. One possible reason is that the diversity of practice contexts in recent years [3] may have further affected the complexity of CR concepts identified in practice [25].

\section{Conclusion}

Our results identified that $\mathrm{CR}$ attributes were "therapeutic thinking processes' and 'professional thinking skills,' the antecedents were 'professional factors' and 'practice factors', and the consequences were 'professional developments' and 'professional attitudes and behaviours.' The resulting framework of clinical reasoning can be the basis for developing pedagogies and assessment of clinical reasoning competence acquisition.

\section{References}

[1] Chapparo C, Ranka J. Clinical reasoning in occupational therapy. In Higgs J, Jones MA, Loftus $\mathrm{S}$ and Christensen N. (Eds.), Clinical reasoning in the health professions 3rd ed. Oxford: Butterworth Heinemann, 2008; 265-78.

[2] Slater DY, Cohn ES. Staff development through analysis of practice. The American Journal of Occupational Therapy. 1991; 45(11): 1038-44.

[3] World Federation of Occupational Therapists. Minimum standards for the education of occupational therapists revised 2016. https://www.wfot.org/resources/new-minimumstandards-for-the-education-of-occupational-therapists2016-e-copy, 2016.

[4] Dent JA, Harden RM, Hunt D, Hodges BD. A practical guide for medical theaters (5th ed). New York, Elsevier, 2017.

[5] Mattingly C, Fleming MH. Clinical reasoning: Forms of inquiry in a therapeutic practice. Philadelphia, Pennsylvania: F. A. Davis, 1994.

[6] Faber RS, Koenig KP. Facilitating clinical reasoning in fieldwork: The relational context of the supervisor and student. In Schell BAB and Schell JW (Eds.), Clinical and professional reasoning in occupational therapy. Philadelphia, Pennsylvania: Lippincott Williams and Wilkins, 2008; 335-367.

[7] Unsworth CA. How do pragmatic reasoning, worldview and client-centredness fit? British Journal of Occupational Therapy. 2004; 67(1): 10-9.

[8] Márquez-Álvarez LJ, Arenillas J, Talavera M, MorunoMillares P. Professional Reasoning in Occupational Therapy: A Scoping Review. Occupational Therapy International. 2019; 1-9.

[9] Unsworth CA and Baker A. Systematic review of professional reasoning literature in occupational therapy. British Journal of Occupational Therapy. 2016; 79(1): 5-16.

[10] Royeen CB, Mu K, Barrett K, Luebben AJ. Pilot investigation: Evaluation of clinical reflection and reasoning before and after workshop intervention. In P Crist (Ed.), Innovations in Occupational Therapy Education. Bethesda, Maryland: American Occupational Therapy Association, 2000; 107-14.

[11] Facione PA. California critical thinking skills test: Form 2000. Millbrae, California: Insight Assessment, 2000.

[12] Maruyama S, Nagatani R. A literature review on the application of the clinical reasoning concept -comparison between pre-2005 and post-2006 in Europe, the United States and Japan- (in Japanese). Japanese Clinical Occupational Therapy Research (rinsyousagyouryouhoukenkyuu). 2016; 3(1): 39-46. 
[13] Young M, Thomas A, Gordon D, Gruppen L, Lubarsky S, Rencic J, Durning SJ. The terminology of clinical reasoning in health professions education: Implications and considerations. Medical Teacher. 2019; 41(11): 1-8.

[14] Kielhofner G. Model of human occupation: Theory and Application (4th ed.). Philadelphia, Pennsylvania: Lippincott Williams and Wilkins, 2008.

[15] Schell BAB. Professional reasoning in practice. In Schell BAB and Gillen G (Eds.), Willard and Spackman's Occupational Therapy 13th. Philadelphia, United States of America, Wolters Kluwer, 2019; 482-97.

[16] Meleis AI. Theoretical nursing: Development and progress (4th ed.). Philadelphia, Pennsylvania: Lippincott Williams and Wilkins, 2007.

[17] Rodgers BL. Concept development in nursing: foundations, techniques, and applications (2nd Ed), Philadelphia, Pennsylvania: W. B. Saunders Company, 2000.

[18] Hupcey JE, Penrod J. Concept analysis: examining the state of the science. Research and Theory of Nursing Practice. 2005; 19(2): 197-208.

[19] Rogers JC. Eleanor Clarke Slagle Lectureship-1983; clinical reasoning: the ethics, science, and art. The American Journal of Occupational Therapy. 1983; 37(9): 601-16.

[20] Moher D, Liberati A, Tetzlaff J, Altman DG. Preferred reporting items for systematic reviews and meta-analyses: the PRISMA statement. PLOS Medicine. 2009; 6(7): e1000097.

[21] Braun V, Clarke V. Using thematic analysis in psychology, Qualitative Research in Psychology. 2006; 3(2): $77-$ 101.

[22] World Federation of Occupational Therapists, Mackenzie L, Coppola S, Alvarez L, Cibule L, Maltsev S, Loh SY, Mlambo T, Ikiugu MN, Pihlar Z, Sriphetcharawut S, Baptiste S, Ledgerd R. International occupational therapy research priorities: A Delphi study. Occupational Therapy Journal of Research: Occupation, Participation and Health. 2017; 37(2): 72-81.

[23] King G, Currie M, Bartlett DJ, et al. The development of expertise in paediatric rehabilitation therapists: The roles of motivation, openness to experience, and types of caseload experience. Australian Occupational Therapy Journal. 2008; 55(2): 108-22.

[24] Maruyama S, Jinbo Y, Saito Y. Pilot study on novice occupational therapists' experience of using narrative reasoning for elderly clients in Japan (in Japanese). Japanese journal of research for the occupational therapy education (sagyouryouhoukyouikukennkyuu). 2019; 18(2): 23-8.

[25] Carrier A, Levasseur M, Bedard D, Desrosiers J. Community occupational therapists' clinical reasoning: identifying tacit knowledge. Australian Occupational Therapy Journal. 2010; 57(6): 356-65. 